\title{
Gamificação e Crowdsourcing no Combate Sustentável ao Aedes aegypti
}

\author{
Ruan Pierre Oliveira ${ }^{1}$, J. Antão B. Moura ${ }^{1}$, Marcelo A. de Barros ${ }^{1}$, Ana Carolina \\ Policarpo Cavalcante ${ }^{2}$, Francisco Barbosa $\mathrm{Jr}^{1}$ \\ ${ }^{1}$ Departamento de Computação - Universidade Federal de Campina Grande (UFCG) \\ CP 10.106 - CEP 58109-900 - Campina Grande - PB - Brasil \\ ${ }^{2}$ Departamento de Enfermagem - Universidade Estadual de Paraíba (UEPB) \\ Caixa Postal 58429-500 - Campina Grande - PB - Brasil \\ \{ruan.pierre.oliveira, carolina.policarpo.cavalcante, jrbarbosa.ufcg\} \\ egmail.com, \{antao, mbarros \}@dsc.ufcg.edu.br
}

\begin{abstract}
The Aedes aegypti mosquito transmits the zika, chikungunya, dengue and yellow fever diseases. The mosquito evolves from eggs that hatch in water pooling anywhere. To fight the mosquito effectively, the population must be educated in its biology, health threats and countermeasures and actively engage in reporting hatcheries to sanitary authorities. This paper proposes an IT system to support such education and combat efforts in a sustainable way. The system incorporates concepts from games, crowdsourcing, georeferenced information systems, computer-based education, mobile computing and entrepreneurship. Preliminary validation studies indicate the system to be an efficient and sustainable training and combat tool against Aedes aegytpi.
\end{abstract}

Resumo. O mosquito Aedes aegypti é o vetor para a zika, chikungunya, dengue e febre amarela. O mosquito evolui a partir de ovos em água parada. $O$ combate ao mosquito seria mais eficaz se a população fosse educada em sua biologia, ameaças à saúde e medidas de prevenção correspondentes e se engajasse em relatar criadouros às autoridades sanitárias. Este artigo propõe um sistema de TI para suportar tal educação e esforços de combate de modo sustentável. O sistema incorpora conceitos de jogos, crowdsourcing, computação móvel e georreferenciada, treinamento por computador e empreendedorismo. Estudos preliminares de validação indicam que o sistema é uma ferramenta eficiente e sustentável para treinamento e combate ao Aedes aegypti.

\section{Introdução}

O mosquito Aedes aegypti é o vetor para transmissão de doenças que representam um problema recorrente de saúde pública com alternância de surto e epidemias de difícil combate: dengue, febres amarela, chikungunya e o zika Vírus, esse último relacionado com a síndrome de Guillain Barré e a microcefalia em bebês (KIKUTE, 2015).

Além de seríssimos problemas de saúde (com risco de morte), o Aedes traz prejuízos econômicos consideráveis à população, em custos de tratamento das doenças, campanhas de prevenção e combate ao mosquito e dos dias parados ou perda de produtividade dos doentes e seus familiares (OLIVEIRA, 2015). De janeiro de 2015 à 
V Congresso Brasileiro de Informática na Educação (CBIE 2016)

Anais do XXVII Simpósio Brasileiro de Informática na Educação (SBIE 2016)

18 de abril de 2016 foram confirmados mais de 740 mil casos de dengue e 4.180 casos suspeitos de microcefalia no Brasil. Apesar desses números alarmantes estudos comprovam uma baixa notificação de focos do mosquito. Isto porque os atuais canais de denúncia de focos - a exemplo do Disk Dengue e Dengue Zap, no caso de Campina Grande, PB que possuem horário de funcionamento restrito, estão sujeitos a erros ou extravio das informações, e apresentam elevado tempo entre a denúncia e ação do agente de campo (BRASIL, 2016).

O combate ao Aedes é uma tarefa difícil. Dessa maneira, parece razoável que o governo se alie à população uma vez que é esta última que tem melhores condições para apontar de forma mais rápida e precisa, focos do mosquito. A prática de utilizar a inteligência coletiva de voluntários colaboradores em uma solução é conhecida por "crowdsourcing" em computação (MARTINS, 2015). Este trabalho trata da aplicação do conceito de crowdsourcing para construção de um sistema de TI para uso no ensino sobre e no combate ao Aedes aegypti. Por outro lado, uma limitação de crowdsourcing é o engajamento dos usuários. Na tentativa de tratar esta limitação, tem-se associado o uso de crowdsourcing à gamificação, utilizando recursos de jogos para engajar colaboradores em ações que tragam benefícios para uma organização (KIKUTE, 2015).

É importante notar que, esses conceitos de crowdsourcing e de gamificação vêm sendo amplamente difundidos também com fins educativos, dirigidos com sucesso a um público-alvo na chamada geração gamer (BRASIL, BARQUE, 2015). De fato, jogos de realidade alternada (KIM et al, 2009), no qual o jogador ora está imerso em um universo virtual ("imaginário") da narrativa (estória do jogo), ora realizando uma missão, como parte do jogo, no mundo real, podem servir para ensinar assuntos ao jogador, em preparação para tarefas no mundo real, e então, retornar para o jogo para obter os resultados da realização da tarefa.

Neste contexto de jogo de realidade alternada e crowdsourcing (aqui, "sistema colaborativo" em tradução livre), o presente artigo propõe uma aplicação móvel, o "AedesBusters", na qual os usuários podem realizar denúncias de focos de Aedes, casos de doenças e agendar visita dos órgãos responsáveis, a um imóvel fechado. A aplicação foi desenvolvida a partir de requisitos dos processos de negócio das Vigilâncias Ambiental e Epidemiológica (VA/VE) de Campina Grande, PB, visando facilitar denúncias através de uma maior conscientização da população por meio de educação continuada, maior adesão de jogadores e eficácia nos processos. Resultados preliminares do uso do AedesBusters oferecem indícios promissores sobre sua eficácia tanto nos aspectos educativos quanto para os indicadores de sucesso das VA/VE e do interesse do mercado em sustentar o jogo.

O restante do conteúdo é organizado em cinco seções. Na seção 2, discutem-se trabalhos relacionados. A seção 3 apresenta a metodologia usada na pesquisa. A seção 4 introduz as funcionalidades do AedesBusters. A seção 5 traz resultados preliminares de estudos de validação. A seção 6 conclui o artigo e comenta sobre trabalhos futuros.

\section{Trabalhos Relacionados}

O uso de crowdsourcing, inteligência e conhecimentos coletivos (de multidões) para resolver problemas em geral e de interesse público, tem sido alvo de artigos científicos e de serviços diversos (OLIVEIRA, 2015). No combate ao Aedes aegypti, podem ser 
V Congresso Brasileiro de Informática na Educação (CBIE 2016)

Anais do XXVII Simpósio Brasileiro de Informática na Educação (SBIE 2016)

citados como exemplos o "Observatório da Dengue" da UFRN, "SC contra a Dengue" da Game Developers de SC, o DengueMap da Ecovec de BH e o "DeuZikaChico" onde se pode informar focos de Aedes, georreferenciados e agendar visitas de agentes.

Nas Filipinas, foi lançado um outro website para vigilância do vetor, com ligações nas escolas públicas do país. O programa inclui um ambiente que atrai os mosquitos para depositarem seus ovos, e no qual uma solução é usada para matar os ovos/larvas. O processo será observado por alunos e professores. Entretanto, há falhas no repasse dos dados, uma vez que os relatórios não são em tempo real, o que pode atrasar decisões (GERONIMO, 2013).

As soluções acima são sites Web, alguns com mapas georreferenciados, mas não, um jogo interativo. Um site Web é um instrumento útil para combate ao mosquito e educação relacionada, mas diferentemente do jogo AedesBusters, fazem coleta passiva de informações, não motivando a multidão tipicamente, através de recompensas a contribuir para solução do problema (KOSONEN, 2014). Os usuários apenas inserem informações no site. Experimentos como aqueles do programa filipino ou o exame de conteúdos em outros sites podem ser uma atividade no mundo real (nas escolas, p.ex.), para educação de jogadores do AedesBusters. A realização destas tarefas educativas pode receber recompensa na forma de pontos ou mudança de nível no jogo. Assim, o sistema proposto nesse trabalho, pode ser visto como uma evolução ou complementação de sistemas de apoio ao combate do mosquito descrito na literatura.

\section{Metodologia}

A pesquisa para o AedesBusters é um estudo quantitativo de abordagem exploratória no qual foi utilizado o procedimento técnico de pesquisa-ação. Segundo Gil (2008) esse estudo é adequado quando há pouco conhecimento sobre a temática a ser abordada e se deseja conhecer, com maior profundidade, o assunto, de modo a torná-lo mais claro.

A pesquisa envolveu estudos exploratórios nas etapas: 1) Pesquisa de campo para conhecer os processos das VA/VE; 2) Identificação e adesão a princípios éticos e legais relativos à pesquisa, além de entrevista semiestruturada com membros das VA/VE para identificar os pontos críticos nos processos de combate ao mosquito; 3) Entrevista com usuários do Disk Dengue; 4) Definição de indicadores de sucesso em parceria com as VA/VE; 5) Definição da arquitetura e apresentação dos screenshots dos componentes móvel e Web do AedesBusters para validação pelas VA/VE e potenciais jogadores; 6) Apresentação da proposta de protótipo para as VA/VE e priorização das funcionalidades; 7) Implementação das funcionalidades priorizadas para o protótipo; 8) Teste-piloto e estudos de validação de face; e, 9) Analise dos dados.

As etapas 4 a 7 têm natureza iterativa, nas quais se apresentam decisões críticas do projeto AedesBusters para aprovação pelos principais stakeholders, profissionais das $\mathrm{VA} / \mathrm{VE}$, e representantes de jogadores. A rigor, a saída da Etapa 9 realimentaria a entrada da Etapa 1, para outros ciclos de ajustes no AedesBusters e nos processos de negócios das VA/VE e mais estudos de validação, além daqueles discutidos neste artigo. Esta retroalimentação, em andamento, mas ainda sem resultados, pode levar vários meses e até anos. Por isto, não é discutida nesse trabalho. 
V Congresso Brasileiro de Informática na Educação (CBIE 2016)

Anais do XXVII Simpósio Brasileiro de Informática na Educação (SBIE 2016)

As fases 1 a 4 permitiram conhecer os principais problemas enfrentados pelas VA/VE no combate ao mosquito. Os indicadores de sucesso foram assim, identificados e priorizados como: i) aumentar o número de notificações; ii) reduzir o número de imóveis fechados; iii) diminuir o tempo de decisão para ação das VA/VE; iv) melhorar a integração entre essas agências; e, v) aumentar os recursos alocados ao combate.

\section{O sistema AedesBusters}

O AedesBusters é um conjunto de ferramentas para intermediar a interação entre a população em geral e agentes das VA/VE e, assim, facilitar o combate ao Aedes. O AedesBusters é essencialmente um Sistema de Informações Georrereferenciadas (GIS) na Web alimentado por um jogo em um dispositivo móvel (Figura 1).

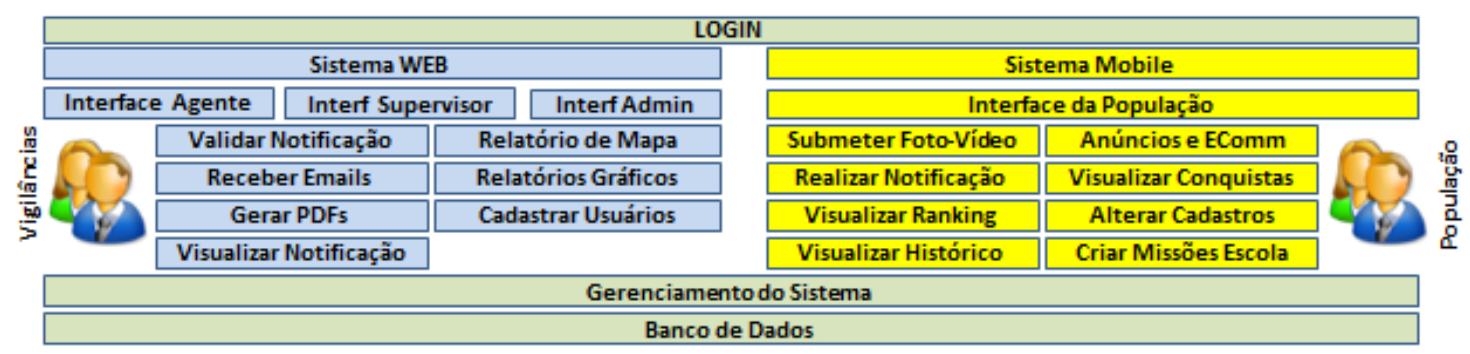

Figura 1. Módulos Arquiteturais e Esquema de Acesso ao AedesBusters

O sistema Web suporta decisões pelas VA/VE. O GIS guarda informações sobre focos do mosquito, imóveis fechados, agendamento de ações ou "missões" (inclusive educativas) atribuídas aos jogadores por agentes das VA/VE ou por eles indicados, como professores, escoteiros, etc. Relatórios gerenciais são produzidos a partir do GIS para apoio a decisões. A alimentação do GIS é feita pelos jogadores seguindo regras e condições no gameplay que roda no equipamento móvel, o qual é mais atraente para alunos e professores em ambientes educacionais (BRASIL, BARQUE, 2015).

Denúncias são comprovadas por fotos ou vídeos carregados no GIS a partir do dispositivo móvel do jogador ou por verificação de agentes próprios ou indicados das VA/VE. A denúncia pode ser feita independente de georreferência (GPS): caso o jogador esteja sem acesso à internet, poderá reportar o foco quando tiver acesso à mesma, localizando o endereço no mapa do aplicativo móvel e confirmando a denúncia. Todas as ações realizadas pelo jogador são passíveis de validação pelas VA/VE antes da atribuição de recompensas correspondentes. $\mathrm{O}$ agendamento de casas fechadas é uma funcionalidade na qual o jogador poderá agendar uma visita de agentes das VA/VE a um imóvel normalmente inacessível para inspeção.

O AedesBusters engloba incentivos intrínsecos e extrínsecos para atrair e manter jogadores. Os intrínsecos incluem motivações pessoais básicas e comunitárias (sentir-se bem ao contribuir para o bem comum) que podem melhorar a patente do jogador ou recompensar conquistas. As extrínsecas, de motivação social como prestígio e status, através do ranking e reconhecimento público, centram-se na estratégia de construir reputação. Outra estratégia é o recrutamento de novos usuários para baixarem e difundirem o aplicativo. Martins (2015) indica que para obter êxito em aplicações de crowdsourcing, a plataforma deve apoiar participantes a recrutarem membros de outras 
V Congresso Brasileiro de Informática na Educação (CBIE 2016)

Anais do XXVII Simpósio Brasileiro de Informática na Educação (SBIE 2016)

comunidades em redes sociais online e esses, por sua vez, difundirem o aplicativo cada vez mais. Escolheu-se a rede social Facebook para difusão do AedesBusters.

A Figura 2 ilustra telas do aplicativo móvel, como o mapa georreferenciado, os ícones para ações no mapa (denunciar focos de Aedes; destruir criadouro; informar sobre doentes com zika, dengue, etc. e agendar visita a imóvel). Na tela do meio, o jogador visualiza seu histórico de conquistas, ranking, pontuações e recompensas tangíveis e intangíveis. A tela à direita mostra uma mudança de nível do jogador, com o acúmulo de pontos. Parte da tela acomoda anúncios, para sustentabilidade do jogo. A sustentabilidade pode ser reforçada com loja virtual, troca de pontos dos jogadores por mercadorias e serviços e comissões em promoções dos patrocinadores.
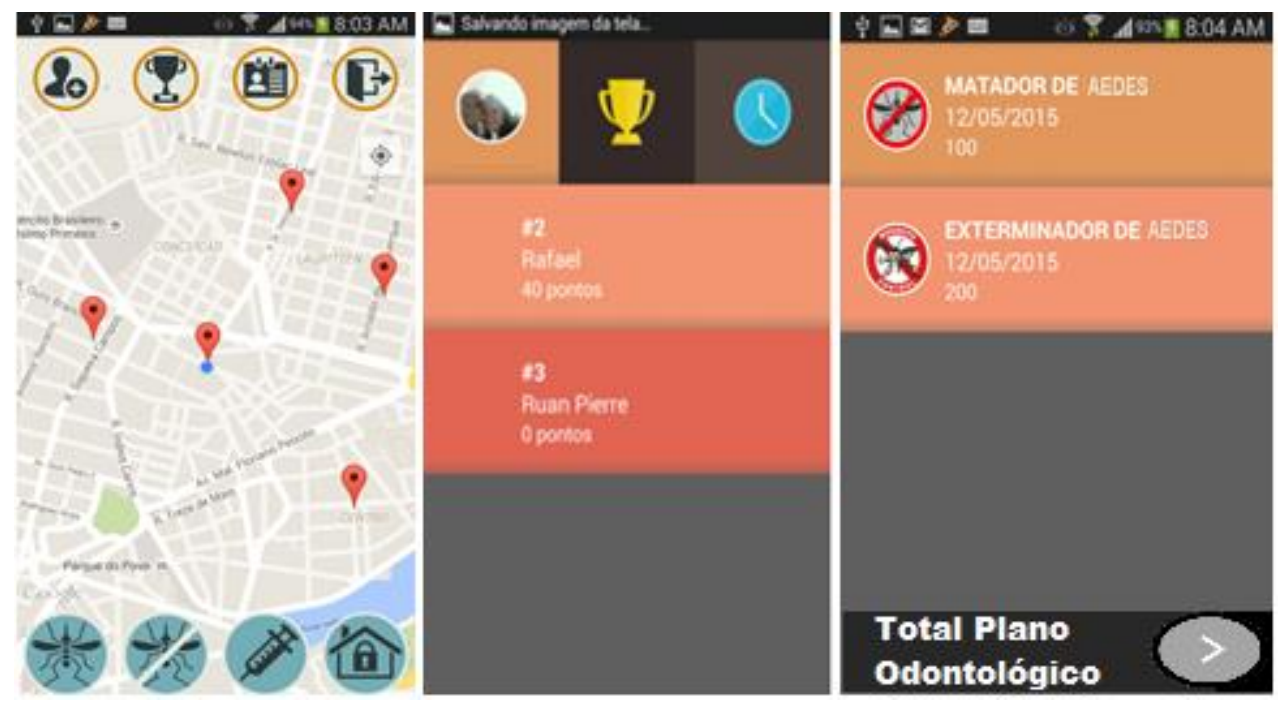

Figura 2. Algumas Telas do AedesBusters com Elementos de Gamificação

A tela básica do componente Web para as VA/VE é um mapa (como na Figura 2), no qual atuam os agentes ou indicados das VA/VE ou jogadores que alcançam certas conquistas no jogo - estes últimos, após endosso pelo supervisor associado, podem atuar como agentes voluntários. Agentes podem receber privilégios apenas para visualizar dados das notificações (localidade, informante e hora); ou, privilégios de supervisor que, além da visualização, podem alterar dados da denúncia, convidar ou endossar agentes voluntários, atribuir missões; ou ainda, privilégios de administrador (técnico) do sistema, para gerenciar o banco de dados (as contas ou cadastros de jogadores, p.ex.) e a produção automática ou sob demanda de relatórios online, com dados inseridos no banco, para apoio a decisões. Note que cada denúncia realizada pela população no aplicativo móvel é automaticamente salva no mesmo banco de dados utilizado pela aplicação Web; assim as VA/VE terão acesso a todas as denúncias realizadas no jogo.

\section{Experimentos de verificação e validação}

Um protótipo do AedesBusters foi desenvolvido espelhado na estrutura da Figura 1. O protótipo não automatizou: o marketplace; agendamento de visitas a imóveis fechados; outras missões (p.ex., direcionamento de ações por agentes das VA/VE); nem incentivos para atrair jogadores. Como os testes foram realizados com poucos jogadores e agentes, estas facilidades foram feitas "por fora" do software. No protótipo, a parte cliente do aplicativo móvel foi implementado para a plataforma Android, usando o GPS nativo do 
V Congresso Brasileiro de Informática na Educação (CBIE 2016)

Anais do XXVII Simpósio Brasileiro de Informática na Educação (SBIE 2016)

Google maps. O servidor foi implementado como um Web Service do tipo Restful usando-se JAX-RS. Para mais detalhes sobre a implementação, como modelo de entidade-relacionamento, casos de uso ou diagrama de classes, favor ver (OLIVEIRA, 2015). O protótipo serviu para testes de verificação e validação pelas VA/VE, por jogadores e empresários, potenciais patrocinadores do jogo.

\subsection{Verificação}

A verificação da arquitetura e da funcionalidade do protótipo AedesBusters foi feita por 26 alunos de vários períodos da Universidade Federal de Campina Grande, UFCG e por 24 agentes das VA/VE, de junho 2014 a junho 2015. Os alunos jogaram o AedesBusters voluntariamente, sem expectativa de ganhos, a não ser aquele do "bem-estar por fazer o bem", não sendo, portanto, influenciados por incentivos, o quais foram omitidos no protótipo. Mesmo assim, $70 \%$ deles concordaram que o jogo motivará denúncias $(27 \%$ ficaram em dúvida e 3\% discordaram), mas apenas $24 \%$ confirmaram disposição em serem "agentes voluntários" (62 \% ficaram em dúvida e 14\% afirmaram que não seriam). A oferta de incentivos, inclusive financeiros, ajudaria a 92\% a mudarem de ideia, declarando-se dispostos a se tornarem agentes voluntários.

Além de opinarem sobre a facilidade de jogar com o protótipo, os agentes profissionais das VA/VE comentaram sobre a utilidade do jogo para as agências. Após usarem o jogo em reunião presencial, os agentes responderam questionário, afirmando unanimemente que esperavam que o jogo contribuísse para diminuir as dificuldades (i) a (v) na seção 3 (Metodologia). Na mesma reunião, os agentes indicaram interesse em conduzir um teste piloto, com experimentos de validação em campo.

\subsection{Experimentos com o piloto para estabelecer validade de face}

Os experimentos nas subseções 5.3 e 5.5 buscam responder Questões de Pesquisa (QP) cuja forma genérica é: Um sistema, como o AedesBusters, baseado em conceitos de gamificação, crowdsourcing contribuirá para o indicador de sucesso "x"? Aqui, o indicador de sucesso " $x$ " é um dos cinco indicadores de sucesso definidos na seção 3 (Metodologia) - i.e., $x \in\{i, i i, i i i, i v, v\}$. QP específica para o uso do AedesBusters no suporte à educação será tratada na subseção 5.4.

Os poucos experimentos realizados, com restritos números de participantes, mas que representam usuários típicos do AedesBusters de cidades do nordeste do Brasil, podem pôr em dúvida a significância estatística dos resultados, limitando a sua generalização. Acredita-se, porém, que os resultados sejam expressivos e úteis, pelo menos para atestar validade de face (LITWIN, 1995). Diz-se que a proposta tem validade de face em determinada aplicação se "o sistema parece" que terá resposta afirmativa à Questão de Pesquisa associada, com mais experimentos e participantes.

\subsection{Validação quanto à eficácia no combate ao Aedes aegypti}

Os experimentos nesta subseção buscam responder às QP relativas aos indicadores $i$ a $i v$ da seção 3 (estudo de validação para a QP do indicador $v$ é alvo da seção 5.5). Os testes foram feitos no bairro Presidente Médici de Campina Grande, PB em julho de 2015. Este bairro foi escolhido pelas VA/VE por ser um dos "mais problemáticos": seu indicador no Levantamento Rápido do Índice de infestação por Aedes aegypti (LIRAa) - 
V Congresso Brasileiro de Informática na Educação (CBIE 2016)

Anais do XXVII Simpósio Brasileiro de Informática na Educação (SBIE 2016)

o qual quantifica o grau de infestação pelo mosquito, chegava então, a 11,5\% (quase o dobro da média da cidade), caracterizando sério risco de surto de dengue (à época, a preocupação com o vírus da Zika era pequena).

A aplicação do protótipo foi feita por 30 voluntários (estudantes, donas-de-casa, professores, policiais e comerciantes), na faixa de idade de 20 a 36 anos, na maioria solteiros, com ensino médio completo, sendo 53,33\% do sexo masculino e 46,66\% do sexo feminino. Todos receberam treinamento no jogo. Dez agentes das VA/VE validavam as notificações pelos jogadores ou atuavam como supervisores, gerando relatórios e realizando o planejamento que serviria de base para decisões.

No experimento, compararam-se os resultados do AedesBusters com aqueles dos canais existentes, Disk Dengue - DD e Dengue Zap - DZ, nos dias 13 a 17 de julho de 2015 (segunda a sexta-feira); os totais de notificações de focos do mosquito pelo DD foi 4; pelo DZ, 5; e, pelo AedeBusters, 38, o que superou o número de cada um dos canais até então disponíveis em pelo menos $760 \%$. Na semana, o AedesBusters aumentou o total de notificações em mais de 520\% (de 9 para 47 - indicando que a situação era pior que apontada pelo LIRAa do bairro). Segundo medições feitas nos processos das VA/VE locais, houve redução de mais de 15 horas no tempo médio de resposta para tipicamente 5 minutos, quando comparado com os tempos médios para decisão/ação usando relatórios dos canais DD e DZ e ou da visitação in loco pelos agentes.

Ao final da semana, foi realizada uma pesquisa com os 30 jogadores, 10 membros da VA/VE e 150 pessoas residentes no bairro considerado. Para cada tipo de respondente foi aplicado um questionário diferente: o dos jogadores focava questões relacionadas ao jogo como, p.ex., se eles estavam motivados e se aprovavam o aplicativo; para os profissionais das VA/VE foi investigado se o aplicativo facilitaria o trabalho diário e se os relatórios seriam úteis; para a população, foram realizadas demonstração e explicação sobre o funcionamento do AedesBusters, com aplicação, logo em seguida, do questionário, para verificar se essas pessoas utilizariam o jogo, se este facilitaria o processo de combate ao Aedes e se eles indicariam o jogo para um parente ou amigo. $90 \%$ dos respondentes das VA/VE indicaram que o jogo "muito facilitaria a integração dos componentes internos da VA/VE" - os mesmos 90\% indicaram que o jogo "muito facilitaria o combate ao mosquito" (os restantes 10\% mencionaram que "pouco facilitaria"). Mais de $90 \%$ dos entrevistados gostaram do jogo e o "recomendariam para um parente ou amigo".

Respostas afirmativas para as QP dos indicadores $i$ ) a $i v$ ) da seção 3 estabelecem validade (de face) do AedesBusters como ferramenta eficaz no combate ao mosquito.

\subsection{Validação como apoio educativo}

Para avaliar o conhecimento adquirido sobre o tema ao se jogar o AedesBusters, os participantes nos experimentos em 5.3 responderam a um questionário. Deles, 76.6\% afirmaram adquirir muito; $16.6 \%$, pouco; e, $6.6 \%$ responderam que não adquiriram nenhum conhecimento. Para avaliar especificamente o potencial do AedesBusters como recurso pedagógico foi realizado um experimento específico. Para testar os benefícios do AedesBusters no ensino-aprendizagem que estimule o empreendedorismo do jogador para explorar possibilidades no contexto mercadológico dos problemas trazidos pelo 
V Congresso Brasileiro de Informática na Educação (CBIE 2016)

Anais do XXVII Simpósio Brasileiro de Informática na Educação (SBIE 2016)

mosquito, principalmente para os negócios do setor $2,5^{1}$, construiu-se um experimento de realidade alternada do tipo blended learning, baseado nas quatro fases da espiral do conhecimento de Nonaka e Takeuchi (1995):

1) Socialização (conversão de tácito para tácito). O professor, agentes das VAVE convidados e estudantes compartilham os saberes sobre o tema Aedes aegypti por meio de observação, imitação, prática em sala de aula e participação em grupos de reflexão sobre um conteúdo a ser estudado. Uma missão é definida pelo professor e pelos agentes, na qual o jogador, além de participar de mini games (testes) sobre o tema ligado ao combate ao Aedes e à prevenção das doenças por ele veiculadas, deve buscar notificar no seu mundo real (escola, lar, ruas...) um fato (criadouro do mosquito, destruição de criadouro...), usando os recursos de comunicação e de registro georreferenciado no aplicativo móvel do AedesBusters.

2) Externalização (tácito para explícito). Os participantes registram seus feitos heroicos em gêneros transmidiáticos de representação do conhecimento tais como fotos, vídeos, áudios de suas leituras de textos e falas e registros textuais de seus relatos por meio de mensagens em redes sociais e descrições no aplicativo AedesBusters.

3) Combinação Os atores, incluindo os professores e alunos, criam conexões desses registros, para elucidar pistas, agregar informações estratégicas sobre o tema estudado e à missão. Geram sistemas de rank, gráficos, mapas e recursos lúdicos para práticas educativas e informações estratégicas para o apoio à tomada de decisão. Com isso, geram novos conhecimentos organizados como novas ferramentas de criação de conhecimento, que assumem o papel de bens de capital, atuando como ferramentas facilitadoras da execução de suas missões na escola e nos demais espaços onde se joga. Dependendo do valor que assumem na evolução da experiência de jogo, tais ferramentas, além de adquirirem valores negociáveis no marketplace do mundo virtual (pontos e moeda virtual do jogo), adquirem valores tangíveis no mundo real.

4) Internalização (explícito para tácito). Os jogadores incorporam ao seu acervo de saberes o conteúdo representado pelo professor tais como o tema de estudo e as missões e os conhecimentos gerados por cada jogador nas missões que realizou.

Um passo inicial de validação do experimento acima foi realizado com a capacitação de 76 professores de escolas públicas de ensinos fundamental e médio em Campina Grande e Sumé, na Paraíba, por meio de convênio da UFCG e UEPB com as secretarias de educação e saúde. Os professores usaram o AedesBusters para criar missões e testar sua eficácia em uma abordagem sócio-construtivista de ensinoaprendizagem junto a seus alunos. Como o experimento com os alunos ainda está acontecendo enquanto se escreve este artigo, apresentamos os resultados da avaliação feita pelos professores. Após o treinamento, os professores responderam a três questões: A) O AedesBusters pode ser usado para capacitar adolescentes na aprendizagem de conteúdos curriculares ligados ao combate ao Aedes aegypti? B) O AedesBusters pode ser usado para capacitação de adolescentes na aprendizagem de outros conteúdos curriculares do $9^{\circ}$ ano do fundamental ao $3^{\circ}$ ano do ensino médio? C) Você está

\footnotetext{
${ }^{1}$ Conceito criado pelo Prêmio Nobel da Paz, Muhammad Yunus, em 2006, que caracteriza um novo paradigma e modelo de gestão das empresas (entidades que pertencem ao Segundo Setor) para gerar impacto social (missão das entidades do Terceiro Setor), e assim criar o Setor 2,5.
} 
V Congresso Brasileiro de Informática na Educação (CBIE 2016)

Anais do XXVII Simpósio Brasileiro de Informática na Educação (SBIE 2016)

satisfeito com o modelo de ensino-aprendizagem proativo (blended learning) do AedesBusters e pode identificar contribuições do mesmo para a educação do $9^{\circ}$ ano do fundamental ao $3^{\circ}$ ano do médio e para o combate a epidemias?

Os resultados são resumidos na figura 3 e demonstram que na opinião dos professores, o AedesBusters e seu modelo proativo de ensino-aprendizagem apoiam tanto a melhoria de processos educacionais quanto o combate ao mosquito Aedes. Em relação à questão $\mathrm{B}$ (uso do AedesBusters para ensinar outros conteúdos), alguns professores demonstraram incerteza alegando dificuldades na criação de missões interdisciplinares envolvendo conceitos da matemática, história, geografia, etc. Esta incerteza indica a necessidade de pesquisa específico para este fim.
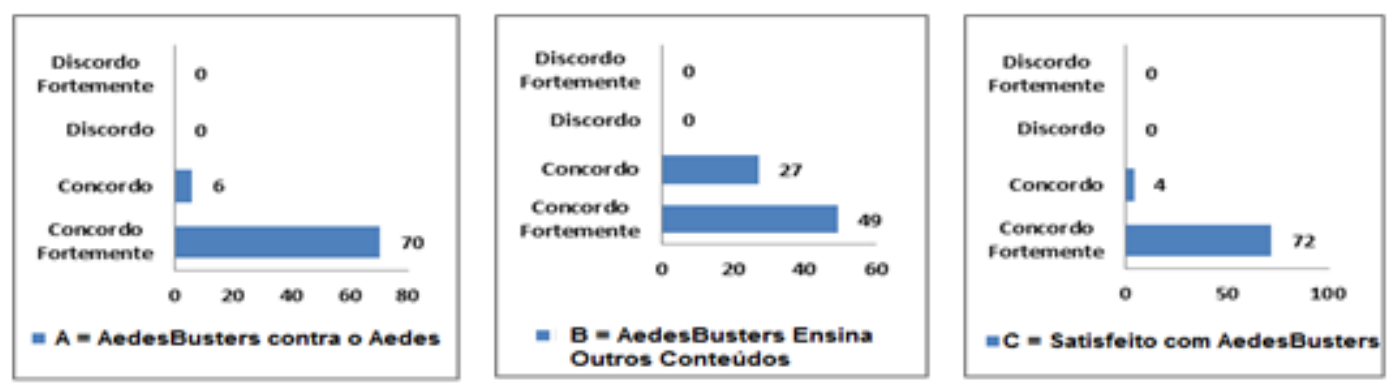

Figura 3: Avaliação realizada com professores da rede pública

\subsection{Validação de sustentabilidade financeira (aspectos de empreendedorismo)}

A sustentabilidade do AedesBusters se apoia nas características de empreendedorismo no marketplace social da proposta e tem a ver com o indicador $v$ da seção 3. A PQ neste estudo passa a ser: O AedesBusters pode gerar fluxos de receita para sua sustentação?

Evidencias do interesse de empresários e comerciantes no AedesBusters foram levantadas por pesquisa em Iguatú, CE, em outubro 2015. Como Iguatú é uma cidade em "situação satisfatória" quanto ao Aedes (LIRAa <1\%), os depoimentos de seus empresários podem ser projetados mais favoravelmente para outras cidades com maior infestação (LIRAa > 1\%). Foram entrevistados 11 empresários dos setores de educação, comunicação (rádios), franquias, lojas de móveis e eletrodomésticos, papelaria, cosméticos, fotografia, promoção de festas e eventos, farmácias, moda masculina, e distribuição de bebidas. Os empresários foram unânimes em acolher o AedesBusters e indicar disposição em participar como anunciantes, oferecendo prêmios ou descontos (de 5 até 30\%) em compras de jogadores que se destacarem no jogo. Em resumo, demonstraram interesse nos aspectos de empreendedorismo do AedesBusters.

Note-se que a sustentabilidade do AedesBusters virá de percentual de negócios e anúncios no marketplace social. Pelos resultados deste experimento de validação, vê-se que QP para o indicador $v$ foi também respondida afirmativamente, levando à validade (de face) da sustentabilidade financeira do jogo proposto. Uso do AedesBusters por empresários aumentará o orçamento alocado ao combate do mosquito.

\section{Conclusões e Trabalhos Futuros}

Este artigo apresentou o AedesBusters, uma proposta inovadora de jogo sério, de realidade alternada, com crowdsourcing, georreferenciamento, computação móvel e empreendedorismo (marketplace social) para a educação e o engajamento da população 
V Congresso Brasileiro de Informática na Educação (CBIE 2016)

Anais do XXVII Simpósio Brasileiro de Informática na Educação (SBIE 2016)

no combate ao mosquito Aedes aegypti, vetor da dengue, zika, chikungunya e febre amarela. O jogo busca sustentabilidade a partir de empreendimentos que o utilizem como um canal de propaganda e negócios. Jogadores serão atraídos por recompensas a conquistas e missões realizadas. As missões podem ser educativas - instruções sobre hábitos do mosquito e práticas para combatê-lo, p.ex., - ou operacionais, na caça e destruição do mosquito. Como tal, o AedesBusters pode ser visto como alinhando recursos de Empreendedorismo e de Informática para a Educação.

Estudos iniciais de validação do AedesBusters produziram indícios promissores de sua utilidade como recurso didático e no combate ao mosquito, melhorando todos os indicadores de sucesso priorizados pela Vigilâncias Ambiental e Epidemiológica parceiras nesta pesquisa. A validação, porém, é preliminar (de face) e necessita de trabalhos continuados para generalizar resultados e conclusões.

Trabalhos futuros devem continuar a investigar aspectos de engenharia de incentivos para complementar prêmios e recompensas existentes; a especificar sistemas de confiabilidade para reduzir fraudes; para desenvolver experimentos educativos que transformem habilidades no jogo em hábitos de combate ao mosquito pela população; e, para conceber novos modelos de negócios para a sustentabilidade financeira. Parece ainda interessante investigar a aplicação de jogos como o AedesBusters, para a solução de problemas como obesidade infantil, gestão de água e violência de gênero.

\section{Referencias}

Brasil (2016). Diretoria de Vigilância Epidemiológica. Situação Epidemiológica da Dengue. Boletim Epidemiológico da dengue - semanas 01 a 15 de 2016. Disponível em: http://www.paho.org. Aceso em 01 de maio de 2016.

Brasil, A. L; Buarque, L.B. "Gamificação Aplicada na Graduação em Jogos Digitais." XXVI Simpósio Brasileiro de Informática na Educação (SBIE 2015).

Geronimo, J.Y. (2013). Crowdsourcing dengue alert. Disponível em: www.rappler.com/nation/31760-nationwide-dengue-vector-surveillance. 14nov2015.

Litwin, M. (1995), How to Measure Survey Reliability and Validity, Sage Publications.

Martins, T. C. M (2015). Crowdsourcing de ideias para inovações no Setor Público. Dissertação (Mestrado Profissional em Administração Pública). 125 p. UFLA.

Kikute, M. (2015) Distribuição espacial e determinantes ecológicos para dengue em comunidade urbana de Salvador, Bahia. (Mestrado em Saúde Coletiva). 70f. UFBA.

Kim, J.; Lee, E.; Thomas, T.; Dombrowski, C. (2009) Storytelling in new media: The case of alternate reality games, 2001-2009.

Kosonen, M. (2014). User Motivations and knowledge sharing in Idea crowdsourcing. International Journal of Innovation Management, Singapore. V.18, n. 5, p. 6-18.

Nonaka, Ikujior \& Hirotaka Takeuchi. The Knowledge-Creating Company: How Japanese Companies Create the Dynamics of Innovation. Oxford: Oxford University Press, 1995.

Oliveira, R.P. (2015). Crowdsourcing e gamificação no combate à Dengue. 2015. 158 f. Dissertação (Mestrado em Ciência da Computação) - UFCG. 